\title{
ATLAS Outreach: on the dissemination of High Energy Physics and Computer Sciences
}

\author{
Arturo Sánchez Pineda* \\ Università di Udine, INFN and ICTP, Italy \\ E-mail: arturosecern.ch

\section{Sascha Mehlhase $*^{\dagger}$} \\ Ludwig-Maximilians-Universität München \\ E-mail: sascha.mehlhasedcern.ch
}

\begin{abstract}
The members of large High Energy Physics (HEP) collaborations come from all over the world. In the case of the ATLAS Collaboration, many of its members come from Latin American, Middle Eastern and Sub-Saharan countries, and so they understand that in many places it can be a challenge to teach experimental HEP. But all members of the collaboration also recognise the massive potential that the always-increasing university student population has, not only concerning fundamental physics but also regarding computing-sciences skills. For that reason, several outreach teams, which include ATLAS, IPPOG, CEVALE2VE members and many other researchers, have been developing and examining free Open Source technologies to release data and to provide effective web-based and offline environments to run, produce, save and share HEP physics analyses. After several years, and with the help of other outreach programmes like the Virtual Visits, ATLAS established an active community that is not just releasing knowledge, data and resources, but genuinely training new physicists who are pursuing advanced studies in experimental HEP right now.
\end{abstract}

7th Annual Conference on Large Hadron Collider Physics - LHCP2019

20-25 May, 2019

Puebla, Mexico

\footnotetext{
* on behalf of the ATLAS Collaboration
}

${ }^{\dagger}$ Speaker. 


\section{Introduction}

The members of the ATLAS Experiment [1,2], at the Large Hadron Collider (LHC) [3] of the European Organisation for Nuclear Research (CERN) [4], have developed an extensive and alwaysevolving outreach programme [5], that -with the collaboration of CERN and other partners- is dedicated to disseminating the physics, the techniques, the results and the implications of the science and engineering that its members produce every day. The outreach activities performed by the ATLAS Collaboration, and many other multinational scientific endeavours are relevant for several reasons. Some of them are moral because resources, funding and support come from the taxpayers of the members countries of the collaboration that directly or indirectly sponsor the programme over 25 years [5]. Another of the crucial reasons is more pragmatic and equally relevant: to ensure continuity of the science over the decades; engaging and training present and future qualified and motivated human resources all over the world. At the same time, ensuring that the knowledge transfer is happening to as many people as possible, and doing so, raising the probabilities that such knowledge can be applied effectively in other areas of science, development and culture.

The above statement sounds ambitious and it is. This is why concrete plans and projects have been expanding to perform such tasks as effectively as possible. One of them has been the release of datasets, educational - web-based and offline - tools and documentation under the so-called ATLAS Open Data project [6, 7, 8, 9]. Another project is the use of video-conferencing technology to "bring" the ATLAS Control Room, the ATLAS detector, its physicists and engineers to the classroom in a real-time conversation between them and primary, high school, university students and their teachers. This project is called the ATLAS Virtual Visit [10]. Nowadays, both projects work together to create an educational resource that every year is used by thousands of students, who -remotely- have a close view of what we do as researchers.

\section{ATLAS Virtual Visits}

The ATLAS Virtual Visits program [10] is a simple but powerful idea: to establish a video conference between ATLAS researchers based at CERN and a university classroom, a school or a public event anywhere in the world with an internet connection. The Virtual Visit (VV) is an online conversation, held in participants' preferred language, between those physicists from ATLAS and students and the general public ready to listen and to ask questions (see Figure 1). An ATLAS VV provides access to the ATLAS Control Room or the ATLAS experimental cavern. So, the participants can get a feeling -as much as the technology allows- of the daily activities of people working on ATLAS detector operations. During this one-hour conversation, the researchers at CERN show the ATLAS Control Room (on the surface) or the ATLAS Detector (100 meters underground) to the participants and explain the physics, technology, operations and challenges of the present experiment and future upgrades (see Figure 2). Tens of thousands of people from all seven continents have participated since the programme launch in 2010. These kinds of video conferences have successfully expanded to events at academic sites, music festivals, conventions and science exhibitions outside the High Energy Physics (HEP) sector [11]. 

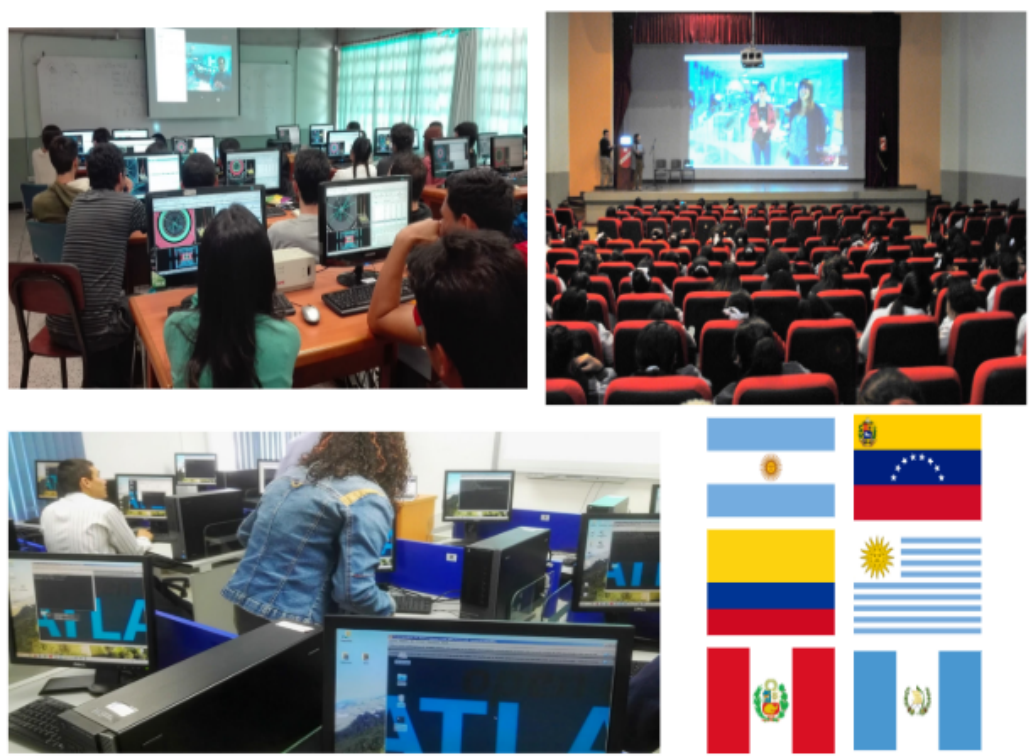

Figure 1: Several events in Latin America (the flags represent some of the countries where educational events were held in 2017 or 2018). On those, the ATLAS Virtual Visits have been used to complement other events like public seminars, IPPOG [12] Masterclasses [13] and training workshops for students.
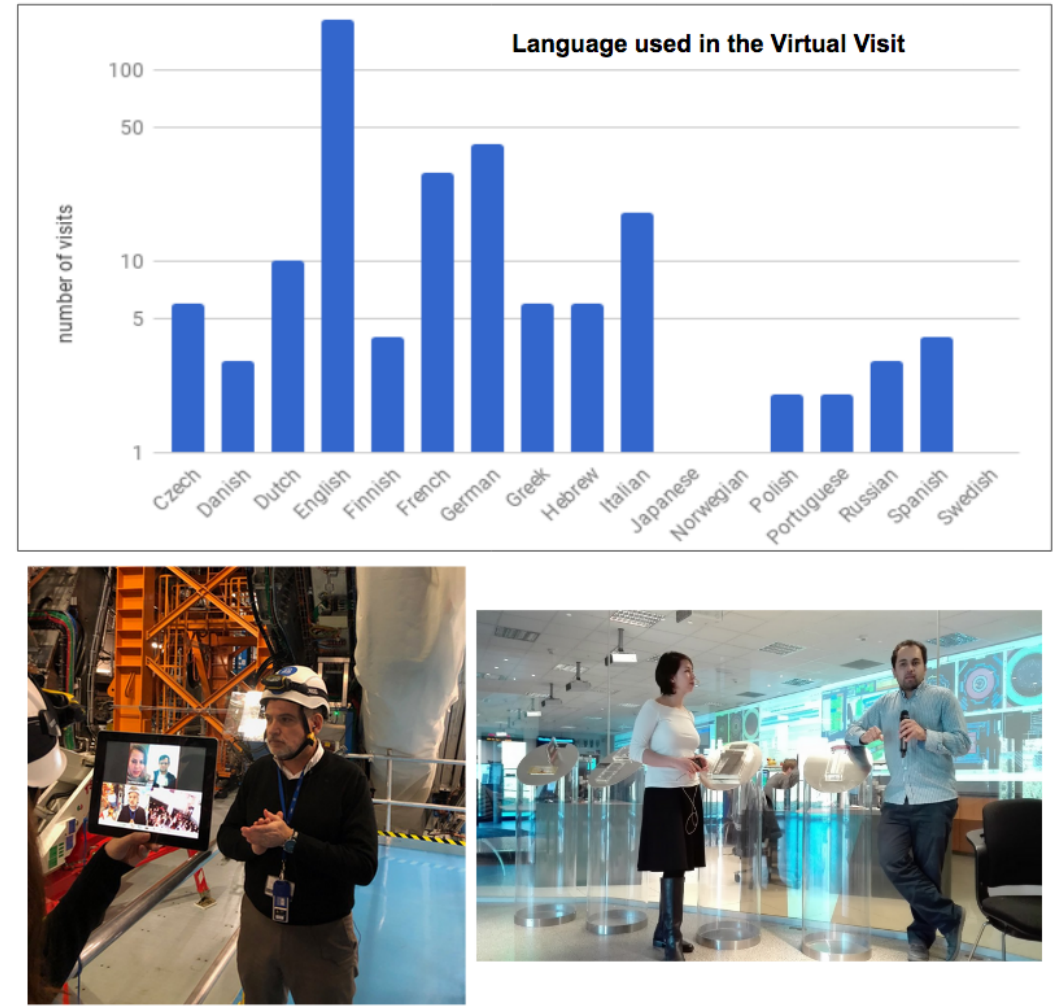

Figure 2: On top: a plot with the languages used to address the participants in ATLAS VV during 2018. On the bottom, two different views of the hosts in an ATLAS VV: underground next to the ATLAS Detector (bottom-left) and at the surface in front of the ATLAS Control Room (bottom-right). 


\section{ATLAS Open Data project}

The ATLAS Open Data project[6, 8, 9] is an effort of the ATLAS Collaboration dedicated to designing a holistic educational programme in HEP for undergraduate and master students all over the world. This effort involves the design, production, testing, validation and analysis of real and simulated Monte Carlo (MC) datasets that will be released to the public following the ATLAS Policy on Open Access with focus on educational and training objectives [14, 15]. Tools and examples and web-based applications are produced and delivered alongside the datasets.

The dataset is designed, tested and constructed to maximise the number of physics processes and analysis examples that can be studied and replicated in a classroom (see Figure 3), while keeping them as small — in terms of digital size — as possible.

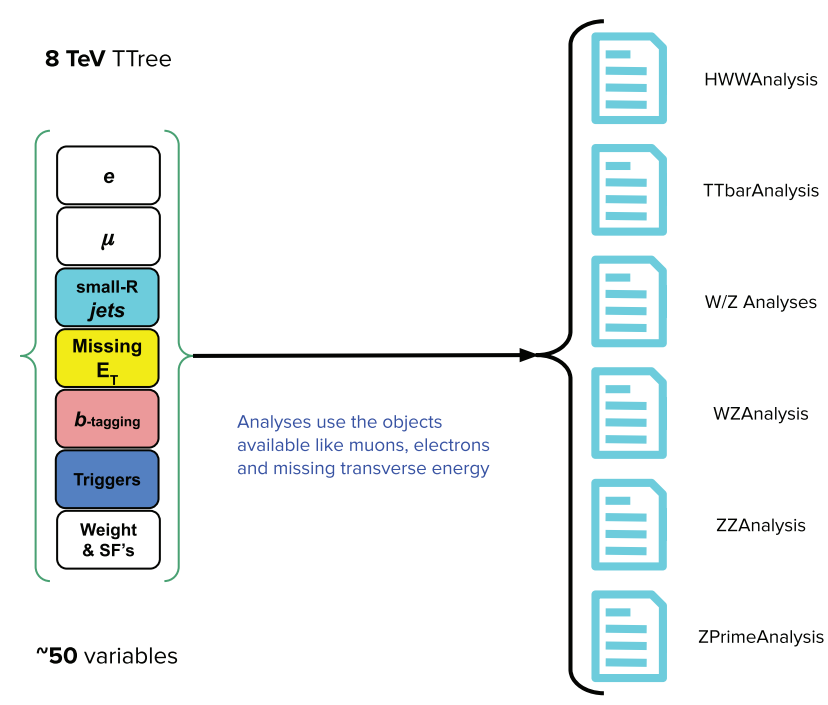

(a) $8 \mathrm{TeV}$ dataset, its content objects and analysis examples

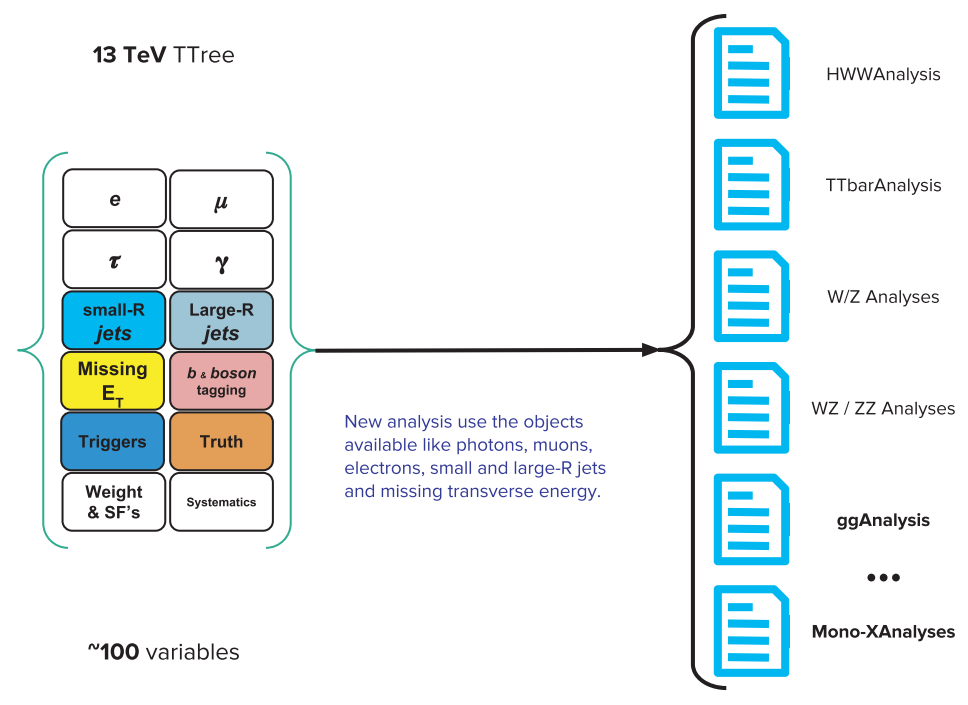

(b) $13 \mathrm{TeV}$ dataset, its content objects and analysis examples

Figure 3: Dataset and analysis examples for the $8 \mathrm{TeV}$ and $13 \mathrm{TeV}$ of the ATLAS Open Data project [16]. 
This way they can fit on and be processed by a commodity computer; as used in labs and by individual students and professors in institutions around the globe. Together with those datasets, software applications, manuals and documentation are produced in ways that can be used with and without constant access to the Internet. In this way, we ensure that, even in not so ideal conditions, students and teachers (see Figure 4) can profit from the dataset and the tools to learn and explore HEP and the relevant computer science. Up to now, ATLAS has released $1 \mathrm{fb}^{-1}$ of real data and several more $\mathrm{fb}^{-1}$, $\mathrm{s}$ of $\mathrm{MC}$ datasets, together with an analysis framework, Jupyter [17] notebooks, dedicated Virtual Machines (VM) and simple web-based and desktop applications in its website [9].

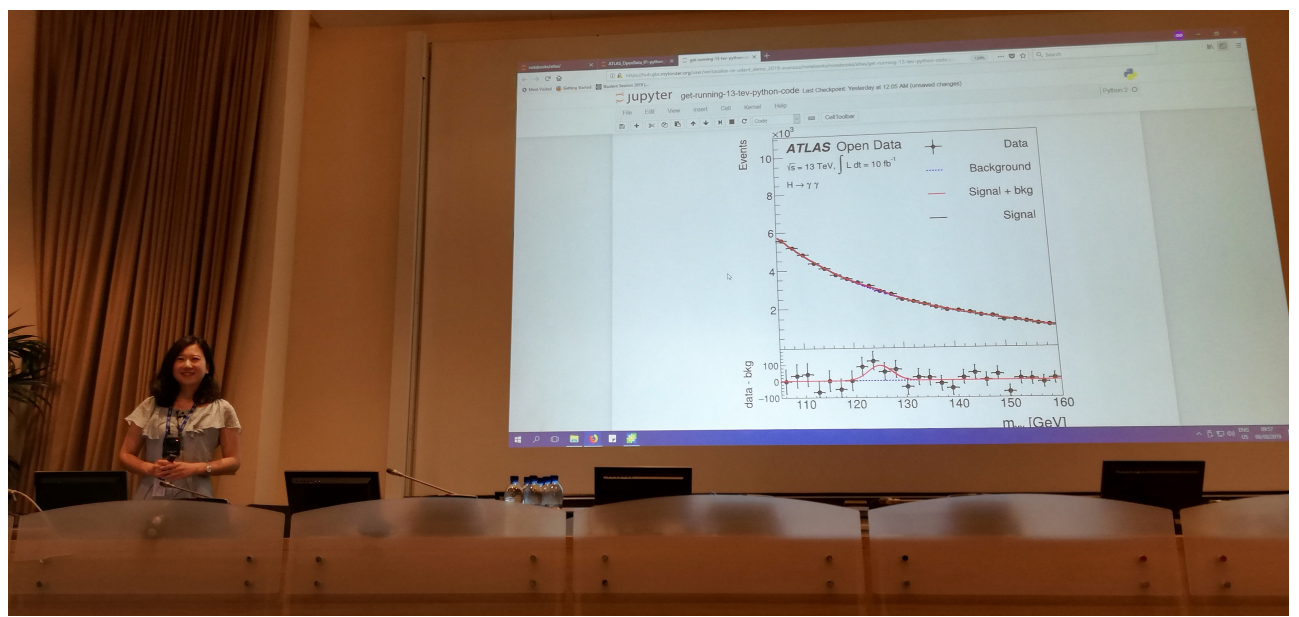

(a) Final ATLAS Open Data Summer Students' report presentation at CERN, August 2019 [18].

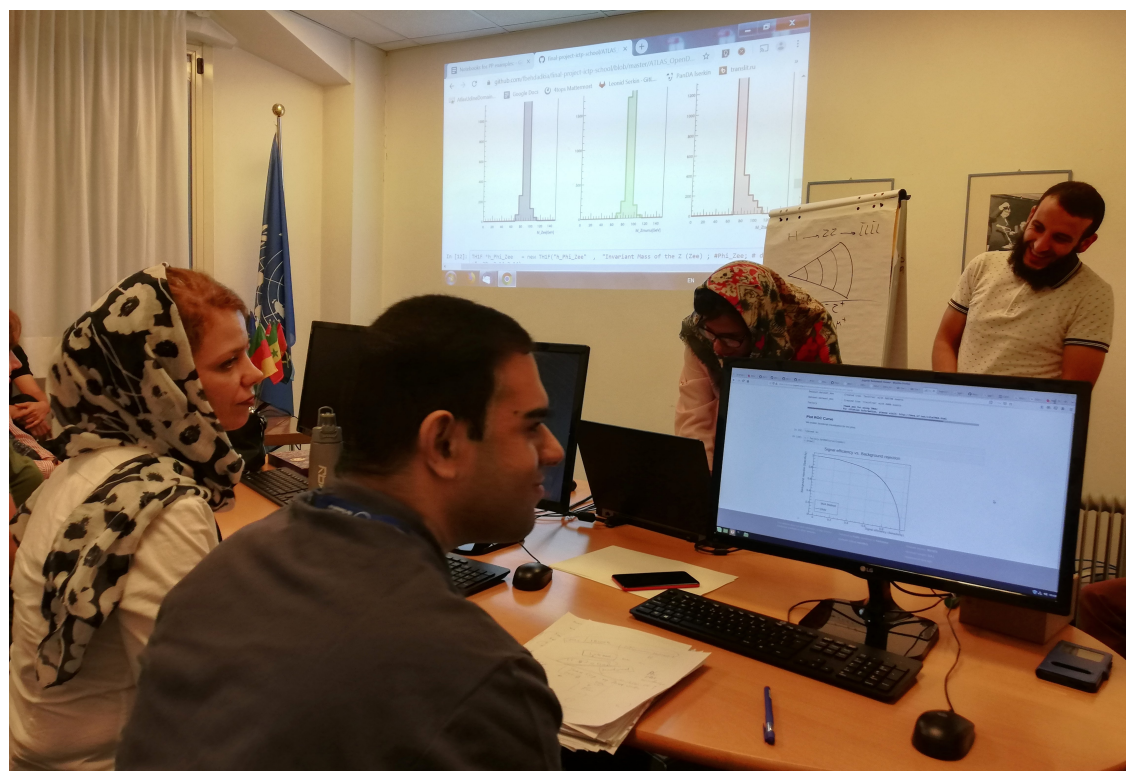

(b) Presentation of exercises of ICTP workshop's students using ATLAS Open Data resources. Trieste, August 2019 [19].

Figure 4: The pictures above show two cases where students and trainers are using ATLAS Open Data educational resources in HEP. In both cases it is possible to see the use of data visualisation web-based tools to (a) demonstrate the final result of a particle physics analysis and (b) discuss the potential of the effect of a particular selection on different MC datasets, including multivariate analysis techniques. 


\section{HEP Outreach collaborations}

In the next sections, some outreach and educational programmes are briefly described. They have been developed independently and currently work in partnerships to enhance their specific activities or to create new ones:

\subsection{IPPOG International Masterclasses}

The ALICE, ATLAS, CMS and LHCb experiments participate actively in the IPPOG [12] International Masterclasses [13]. Each year more than 14000 high school students in about 50 countries come to one of about 220 nearby universities or research centres for one day to unravel the mysteries of particle physics in a simple, practical and engaging session of data analysis. Lectures from active scientists give insight in topics and methods of basic research on the nature of matter and forces, enabling the students to perform measurements on real data from particle physics experiments themselves. At the end of each day, like in international research collaboration, the participants join a video conference for discussion and combination of their results, moderated by physicists in research centres in the Americas and Europe.

\subsection{CEVALE2VE}

The Centro de Altos Estudios de Altas Energías (CEVALE2VE) [20] is a virtual research and learning community created to support the new generation of Venezuelan researchers in HEP. It also attempts to contribute to the knowledge transfer of fundamental physics and the regional modernisation of university education with the promotion of scientific communities in Latin America. The project organisation involves several academic institutions in Venezuela, Colombia, Peru, Guatemala, Ecuador and Mexico, to reach a broad regional audience. Although geographically scattered in different academic institutions of Europe and North America, CEVALE2VE integrates a group of Venezuelan researchers currently involved in projects related to the LHC. Its main goal is to stimulate and widen regional physics postgraduate education and research, the first step of which has been the implementation of the online university course "Introduction to Particle Physics" [20]. The main objective is to develop a virtual research and learning community to promote education and research in the fields of HEP and computer sciences in the Latin American scientific community [21] (see Figure 5). The CEVALE2VE activities can be summarised as: Education and interest in research: positively influence and stimulate physics students' interest in HEP research.

- Awareness of opportunities: educate and invigorate the students with further study opportunities in physics and possible career paths in research.

- Create networks and collaborations: between the European and North American institutions and research centres with their partner universities in Latin America.

- Modernisation of education and training: through the use and development of a web platform with e-learning open access tools.

- Policymaking: working towards formalising the involvement of Latin American institutions in HEP experiments. 

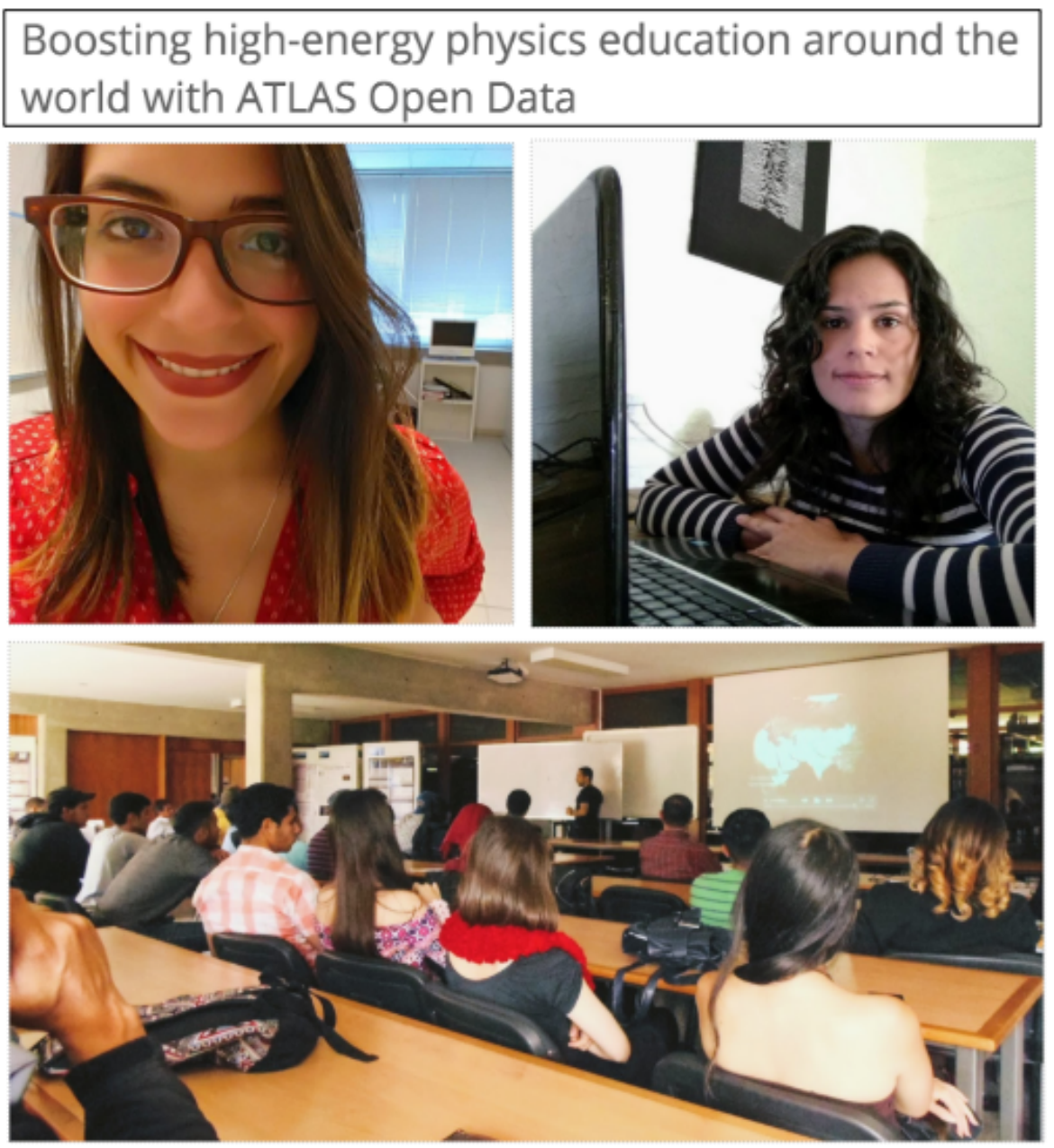

Figure 5: "Boosting high-energy physics education around the world with ATLAS Open Data" [21].

\subsection{ICTP Physics Without Frontiers}

The ICTP [22] Physics Without Frontiers (PWF) [23] programme is an outreach set of activities designed by different researchers, especially in the developing world, under the guidance and supervision of ICTP coordinators. This is a programme that designates resources and support to approved applications coming from an inclusive community of researchers, professors and science communicators, among others. The PWF programme works to inspire, train and motivate physics and mathematics university students worldwide, to help build the next generation of scientists. Each project is unique, developed with the country's specific needs in mind.

PWF organises projects working with volunteer scientists, who are $\mathrm{PhD}$ students, postdoctoral researchers, or lecturers from all over the world passionate about promoting physics and mathematics. PWF has worked with over 5000 students worldwide in 17 different countries [23]. 


\subsubsection{Physics Without Frontiers projects}

- University Courses: PWF partners with physics and mathematics departments that are unable to teach specific courses due to a lack of resources. The programme arranges an expert to visit the university to teach the course and where possible train local researchers and teachers.

- Schools: PWF organise pedagogical schools at universities for local undergraduate and master students across the country. The programme aims to expose and train the students in research fields they lack access to.

- Roadshows: Roadshows are organised by groups of young researchers, passionate about advocating their research field back in their home [24]. The roadshow centres around intensive one-day activities in universities across the host country, in addition to outreach and public events (see Figure 6).

The PWF programme has the final aim of breaking down barriers in accessing physics and mathematics research. This means connecting with all students and scientists worldwide, and with those from less represented groups in physics and mathematics within countries, due to factors such as gender, economic background, ethnicity, or those who may lack access due to geographic barriers or isolation within a region.

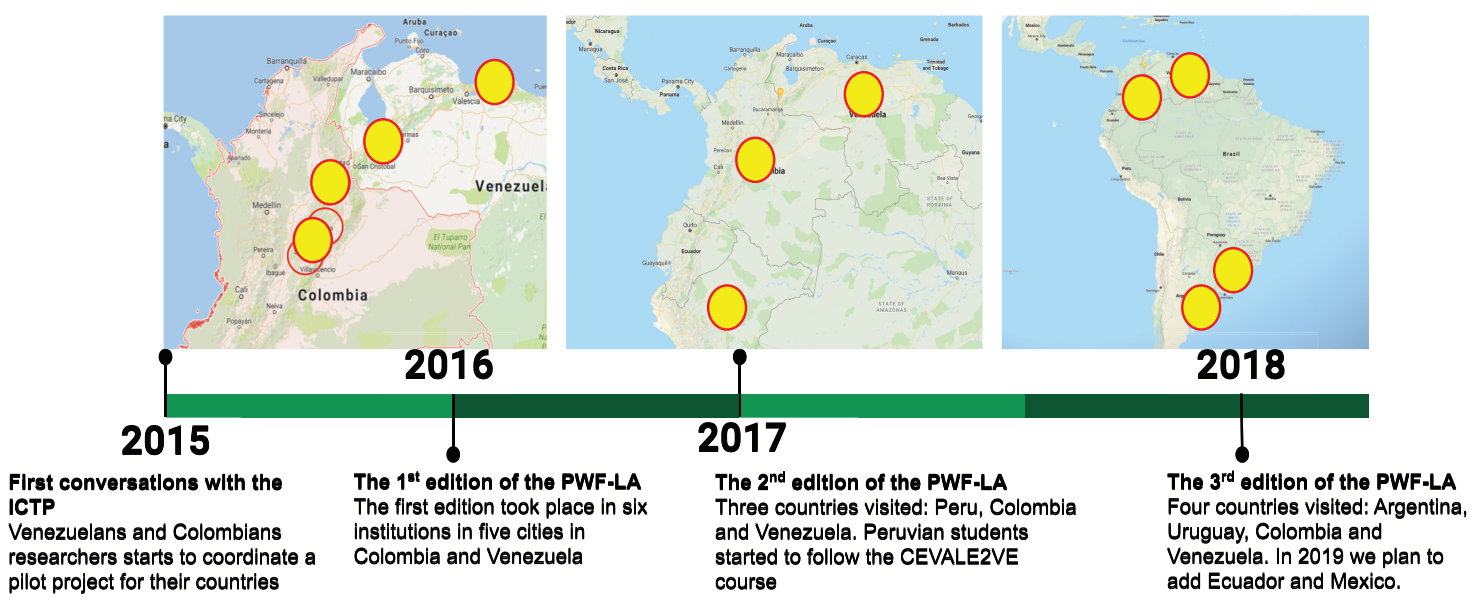

Figure 6: A summary of the ICTP PWF and CEVALE2VE programme in Latin America. The set of maps shows the evolution of the roadshows in multiple cities in the continent [24].

\section{Joint Efforts \& Summary}

Promoting and performing HEP's educational programmes, training and outreach in a multicultural and globalised environment is a constant challenge, and a source of opportunities to innovate and to collaborate between individuals, institutions, programmes and countries to take the best of their efforts and join them, creating useful, broad and maintainable projects in the long term. 
The ATLAS Outreach group, in particular, the Open Data and Virtual Visits programmes have joined resources and efforts to create activities that involve people from many educational, cultural, geographical and institutional backgrounds, improving the knowledge transfer and the exchange of ideas and the development of human resources into the HEP sector. Other partnerships have been established with external outreach groups, making the impact of such programmes even larger. Nowadays, outreach communities like IPPOG, CEVALE2VE, ICTP PWF and many individuals are executing successful projects that invite even more researchers and institutions to get involved [25, 26, 27]. This positive evolution in open access resources, activities and events is increasingly getting the attention and support of the policymakers, and large scientific collaborations that are evident in dedicated sessions in important conferences like the one that this article is intended to document. The overall observation is that the different groups have been finding ways to enhance the education of diverse communities, thanks to the collaboration and constant feedback among them. No surprise there, this is how science moves forward also.

\section{References}

[1] ATLAS Collaboration, The ATLAS Experiment at the CERN LHC. 2008 JINST 3 S08003.

[2] The ATLAS Collaboration at LHC. https://atlas.cern

[3] The Large Hadron Collider (LHC). https://home.cern/science/accelerators/large-hadron-collider

[4] The European Organisation for Nuclear Research (CERN). https://home.cern

[5] ATLAS Collaboration, ATLAS: A 25-Year Insider Story of the LHC Experiment Chapter 11: ATLAS Collaboration: Life and its Place in Society

Advanced Series on Directions in High Energy Physics: Volume 30, 2019. https://doi.org/10.1142/9789813271807_0011

[6] ATLAS Collaboration, Review of ATLAS Open Data Dataset. ATL-OREACH-PUB-2016-001. https://cds.cern.ch/record/2203649/.

[7] ATLAS Collaboration, Review of ATLAS Open Data 8 TeV datasets, tools and activities. ATL-OREACH-PUB-2018-001 https://cds.cern.ch/record/2624572/

[8] Katarina Anthony, Article: Explore LHC data on new ATLAS educational platform. July, 2016. http://cern.ch/go/MSN7

[9] The ATLAS Open Data web portal. http://opendata.atlas.cern

[10] Steven Goldfarb, Sahal Yacoob on behalf of the ATLAS Collaboration, The ATLAS Virtual Visits project.. Nucl.Part.Phys.Proc. $273-275$ (2016) 2578-2580

[11] ATLAS Connects with Science Fest Visitors. http://atlas.cern/tags/virtual-visits

[12] The International Particle Physics Outreach Group (IPPOG) web portal. http://ippog.org

[13] The IPPOG Masterclasses web portal. https://physicsmasterclasses.org

[14] The ATLAS Data Policy. http://opendata.cern.ch/record/413/files/ATLAS-Data-Policy

[15] Arturo Sánchez Pineda, CERN Courier: Preserving the Legacy of Particle Physics. Page 33. March-April 2019.

https://cerncourier.com/wp-content/uploads/2019/07/CERNCourier2019MarApr-digitaledition.pdf 
[16] ATLAS Collaboration, Proposal for an ATLAS endorsed 13 TeV data set for Outreach Purposes. The ATLAS Collaboration. https://cds.cern.ch/record/2679254

[17] The Jupyter Open Source project. https://jupyter.org/

[18] 2019 CERN Summer Student for the ATLAS Open Data group's final presentation. https://cds.cern.ch/record/2685132

[19] The 2019 ICTP "The CODATA-RDA Research Data Science Advanced Workshops on Bio-informatics, Climate Data Sciences, Extreme Sources of Data and Internet of Things(IoT)/Big-Data Analytics". http://indico.ictp.it/event/8847

[20] Arturo Sánchez Pineda, The CEVALE2VE case. ATL-OREACH-PROC-2017-001 https://cds.cern.ch/record/2241903/

[21] Arturo Sánchez Pineda, Article: Boosting high-energy physics education around the world with ATLAS Open Data. July, 2018. http://cern.ch/go/7nv8

[22] the Abdus Salam International Centre for Theoretical Physics (ICTP) web portal. https://www.ictp.it/

[23] The ICTP Physics Without Frontiers (PWF) web portal. https://www.ictp.it/physics-without-frontiers.aspx

[24] Arturo Sánchez Pineda, Article: Physics Outreach: Colombia and the ICTP Physics Without Frontiers + CEVALE2 VE. November, 2018. http://cern.ch/go/St6p

[25] M. R. Di Domenico Franco, Reconstruction of the invariant masses of bosons of the Standard Model using public data from ATLAS Open Data. CERN-THESIS-2017-239. https://cds.cern.ch/record/2293251/

[26] I. Garcia, Perspectives and Evaluation of Dark Matter production in association with a light quark, a heavy quark (b-quark) or an electroweak boson in particle colliders at a centre-of-mass energy of $\boldsymbol{8} \boldsymbol{T e V}$. CERN-THESIS-2017-217. https://cds.cern.ch/record/2291838/

[27] M. O. Evans, Enabling Open Science with the ATLAS Open Data project at CERN. CERN-THESIS-2018-099. https://cds.cern.ch/record/2630961/ 